Document downloaded from:

http://hdl.handle.net/10251/140908

This paper must be cited as:

Monsoriu Serra, JA.; Gimenez Valentin, MH.; Riera Guasp, J.; Vidaurre, A. (2005).

Measuring coupled oscillations using an automated video analysis technique based on image recognition. European Journal of Physics. 26(6):1149-1155.

https://doi.org/10.1088/0143-0807/26/6/023

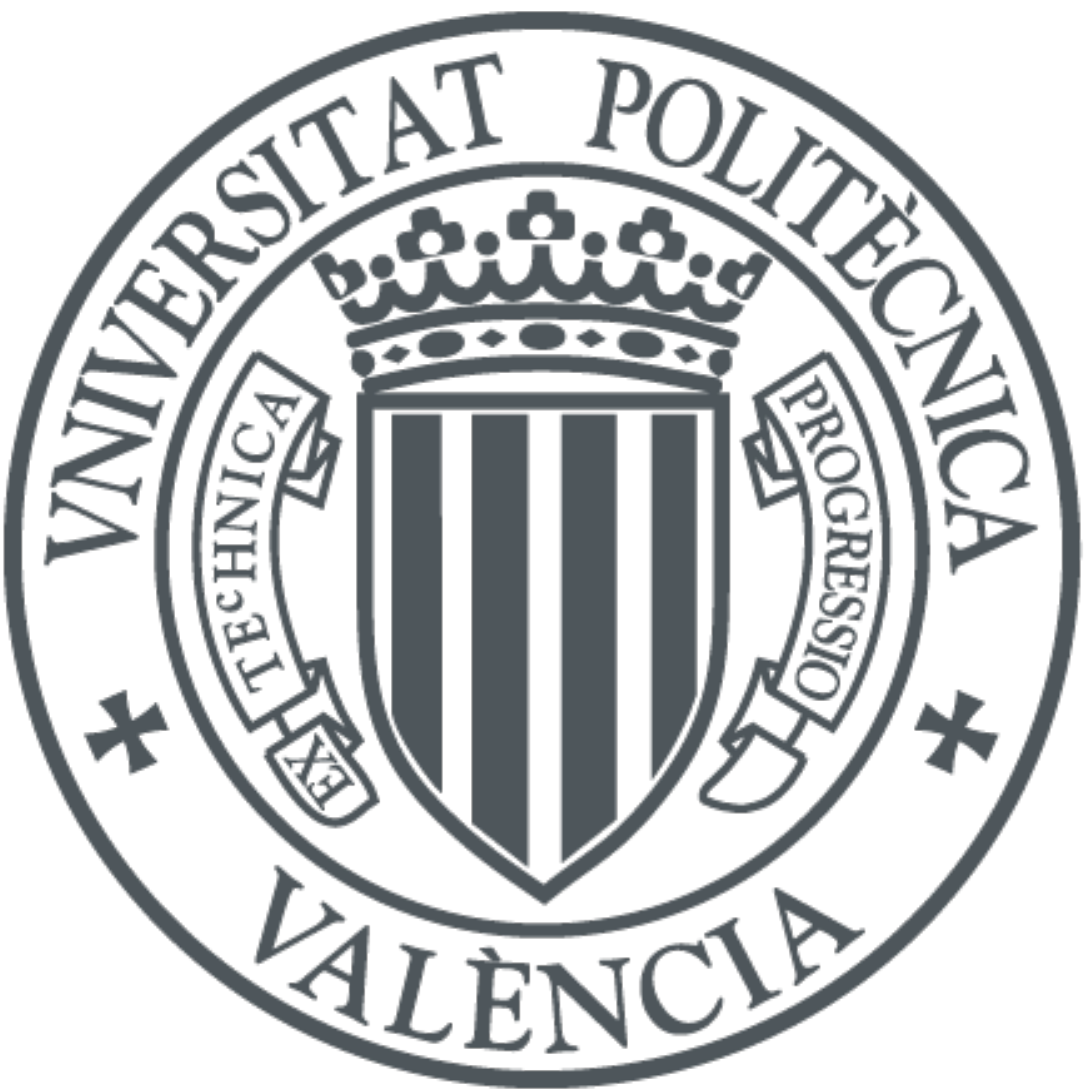

The final publication is available at

https://doi.org/10.1088/0143-0807/26/6/023

Copyright IOP Publishing

Additional Information 


\title{
Measuring coupled oscillations using an automated video analysis technique based on image recognition
}

\author{
Juan A. Monsoriu*, Marcos H. Giménez, Jaime Riera, and Ana Vidaurre \\ Departamento de Física Aplicada, Universidad Politécnica de Valencia, \\ E-46022 Valencia, Spain
}

\begin{abstract}
The applications of digital video image to the investigation of physical phenomena have increased enormously in recent years. The advances in computer technology and image recognition techniques allow the analysis of more complex problems. In this work we study the movement of a damped coupled oscillation system. The motion is considered as a linear combination of the two normal modes, i.e., the symmetric and antisymmetric modes. The image of the experiment is recorded with a video camera and analyzed by means of software developed in our laboratory. The results show a very good agreement with the theory.
\end{abstract}

KEYWORDS: Image processing, physical measurements, coupled oscillations

PACS numbers: 01.50.Lc, 42.30.Sy

*E-mail: jmonsori@fis.upv.es 


\section{INTRODUCTION}

Digital techniques have shown to be a very useful tool for the analysis and understanding of physical concepts [1-4]. In particular, digital simulations allow the visualization of the physical process. In this way, the students can improve their knowledge of the real physical process through a model rather than through its oral explanation [5-7]. Unfortunately, many simulations used in teaching physics only display the end product but not contribute to the understanding of the physical phenomena. When using simulations the teacher must be sure that they serve to increase the students' understanding [8].

Video analysis was initially used to investigate simple problems related to kinematic concepts $[9,10]$. The development of point-and-click tools such as VideoPoint $^{\mathrm{TM}}$ and VideoGraph ${ }^{\mathrm{TM}}$ allows the quantitative analysis of more complex problems $[11,12]$. Recent works $[13,14]$, confirm the interest of video analysis applied to more advanced concepts in physics. It has also been proposed as a remote and non invasive technique in the area of system identification involving non-linear characteristics of mechanical and structural systems [15].

However, the application of video analysis to the investigation of increasingly complex problems involves working with a great number of images. In order to reduce errors and avoid the excessively tedious process of image processing, new automatic image recognition techniques have been developed $[15,16]$. In this work we analyze a mechanical system consisting of two gliders mounted on an air track. Between the gliders there is a spring, as well as between the gliders and the fixed extremes of the air track. Therefore, the system consists of two coupled oscillators. Recently, a simple method to observe normal modes in coupled oscillators has been proposed [17]. This method consists of applying a frequency dependent force to the system and using 
resonance to excite each mode separately. However, it doesn't allow the analysis of a coupled movement generated by an arbitrary combination of the two normal modes. This latter case can be studied using video analysis technique that gives the position of both gliders every 0.04 seconds. With this technique, the student not only obtains the quantitative results, but thanks to the visual display of the process he/she also gains a better understanding of the physical phenomenon. We have used standard linear correlation [18] as the basis for the detection technique. It is same technique as that used to analyze the movement of a body at constant speed and constant acceleration [16]. In this case, the positions of more than one object on each image need to be obtained. The detection technique has revealed also suitable for this task, opening a new field of applications.

This paper is organized as follows. The experimental design and the measurement techniques are shown in Section II. The theoretical basis of a system of coupled oscillators is described in Section III. The experimental results, fitting and discussion are explained in Section IV. Finally, concluding remarks are presented in Section V.

\section{EXPERIMENTAL PROCEDURE}

The experimental arrangement consists of two identical gliders connected to each other by a spring and to the confining walls by other two identical springs. The gliders are mounted on an air track in which friction can be regulated through the air flow (see Fig. 1)

The digital camera used in the experiments was a Panasonic NV-DS15EG, with an exposure time of $1 / 2000 \mathrm{~s}$ and a rate of $25 \mathrm{frames} / \mathrm{s}$ providing a time resolution of $0.04 \mathrm{~s}$. The camera was placed with its axis perpendicular to the movement direction, at 
a distance of $1.5 \mathrm{~m}$. The video system was PAL (Phase Alternation by Line) that produces $720 \times 576$ pixel images. We performed the analysis on $512 \times 256$ pixel windows, thus obtaining a good balance between information accuracy and computational cost.

For the localization of the moving objects, we considered each frame as an input scene, $f(x, y)$, and used a detached image as the filter for the particular object of interest, $h(x, y)$. The correlation function between these functions is given by [16,18]:

$$
g(x, y)=\int_{-\infty}^{\infty} \int_{-\infty}^{\infty} f\left(x^{\prime}, y^{\prime}\right) h\left(x^{\prime}-x, y^{\prime}-y\right) d x^{\prime} d y^{\prime}
$$

The maximum of the correlation function (see Fig. 2) corresponds to the location where the scene is most similar to the target object.

When working with digitized images, correlation function involves double integrations that require much computation time. However, the process can be substantially simplified by using the properties of Fourier transforms. It can be easily proved that [19]:

$$
g(x, y)=F^{-1}\{F\{f(x, y)\} F\{h(-x,-y)\}\} .
$$

In this way, the correlation function can be calculated relatively rapidly using the direct and inverse Fourier transforms based on Fast Fourier Transform (FFT) algorithms. The image recognition software has been developed in VisualBasic and runs on Windows platforms. Two extreme positions of the gliders were tested in order to know if the system resolution allows the location of both gliders. Fig. 2 shows the correlation function at these positions, where the distance between the gliders is maximum (Fig. 2a) and minimum (Fig. 2b). In all cases, the system resolution allowed the location of both gliders. 


\section{THEORETICAL BASIS}

Let us consider the system shown in Fig. 1 consisting of two equal masses $m$ connected to each other by a spring with elastic constant $\mathrm{k}_{\mathrm{i}}$, and to a fixed point by similar springs with elastic constant $k_{\mathrm{o}}$. The system is horizontally aligned; let us now consider one-dimensional motion along the line connecting the masses. We define the coordinates, $x_{1}$ and $x_{2}$, as the displacement to the right from equilibrium for gliders 1 and 2 respectively. If we apply Newton's law to each mass, we find the following equations of motion:

$$
\begin{aligned}
& m \ddot{x}_{1}=-k_{o} x_{1}-k_{i}\left(x_{1}-x_{2}\right)-c \dot{x}_{1} \\
& m \ddot{x}_{2}=-k_{o} x_{2}-k_{i}\left(x_{2}-x_{1}\right)-c \dot{x}_{2}
\end{aligned}
$$

where $c$ is the coefficient of the velocity dependent damping force. It is possible to uncouple these equations by introducing a new set of coordinates $q_{1}=\left(x_{2}+x_{1}\right)$ and $q_{2}=\left(x_{2}-x_{1}\right)$. Then:

$$
\begin{aligned}
& m \ddot{q}_{1}+c \dot{q}_{1}+k_{o} q_{1}=0 \\
& m \ddot{q}_{2}+c \dot{q}_{2}+\left(2 k_{i}+k_{o}\right) q_{2}=0
\end{aligned}
$$

Each of the two independent equations corresponds to the motion of a damped harmonic oscillator. The solution of these equations is:

$$
\begin{aligned}
& q_{1}=A_{1} e^{-t / \tau} \sin \left(\omega_{1} t+\phi_{1}\right) \\
& q_{2}=A_{2} e^{-t / \tau} \sin \left(\omega_{2} t+\phi_{2}\right)
\end{aligned}
$$

where $\omega_{1}=\sqrt{\omega_{01}^{2}-\gamma^{2}}$ and $\omega_{2}=\sqrt{\omega_{02}^{2}-\gamma^{2}}$ are the angular frequency corresponding to each normal mode; $\phi_{1}$ and $\phi_{2}$ represent the initial phase of each oscillating mode, $\tau=\gamma^{-1}$ is the relaxation time, and $\gamma=c /(2 m)$ is the damping coefficient. If this coefficient is negligible, the angular frequencies are $\omega_{01}=\sqrt{\frac{k_{o}}{m}}$ and $\omega_{02}=\sqrt{\frac{\left(2 k_{i}+k_{o}\right)}{m}}$. 


\section{RESULTS AND DISCUSSION}

\section{A. Normal modes.}

First, we tried to reproduce the oscillating normal modes separately. For this end, the system was excited in the appropriate way. In Fig. 3 the coordinates $q_{1}$ and $q_{2}$ are plotted as a function of time. Hereafter, the corresponding uncertainty bars of the data are not shown in this kind of representation because they have the same size of the symbols. Observe in Fig. 3 that the antisymmetric mode is almost negligible. This is because the initial displacement of the masses $\left(x_{01}=2 \mathrm{~cm}, x_{02}=2 \mathrm{~cm}\right)$ is the same, and this favors the symmetric mode in which both masses move in the same direction. From the fitting of the curve we obtain $A_{1}=58.0(0.4)$ pixels, $\omega_{01}=9.11(0.02) \mathrm{rad} / \mathrm{s}$, and $\phi_{1}=-$ $3.51(0.02) \mathrm{rad}$ where the number in parenthesis is the numerical value of the combined standard uncertainty expressed in the same unit of the quoted result. The parameters and their uncertainties were obtained using standard least squares method.

Fig. 4 shows the corresponding results when the initial displacements favor the antisymmetric mode $\left(x_{01}=-2 \mathrm{~cm}, x_{02}=2 \mathrm{~cm}\right)$. In this case, the difference in the presence of both modes is not so remarkable as in the previous situation, maybe due to noise effects. From the fitting of the experimental data we obtain $A_{2}=35.6(0.4)$ pixels, $\omega_{02}=16.86(0.04) \mathrm{rad} / \mathrm{s}$, and $\phi_{2}=-3,04(0.02) \mathrm{rad}$. The theoretical relationship between the angular frequencies of the normal modes obtained from the elastic constants of the system, $k_{i}=55.3(0.9) \mathrm{N} / \mathrm{m}$ and $k_{o}=45.9(0.6) \mathrm{N} / \mathrm{m}$, is $\frac{\omega_{02}}{\omega_{01}}=\sqrt{1+2 \frac{\mathrm{k}_{\mathrm{i}}}{\mathrm{k}_{\mathrm{o}}}}=1.846(0.046)$. This result is in a very good agreement with the value obtained in the video-analysis, $\left.\frac{\omega_{02}}{\omega_{01}}\right|_{\exp }=1.851(0.006)$. In both cases, the value of the combined standard uncertainty 
has been determined through the law of propagation of uncertainty [20].

\section{B. Movement without friction.}

If the air flow of the air track is high enough, the damping coefficient is very small. Figure 5 shows the normal modes in the case of negligible damping coefficient, the initial position of the masses being $x_{01}=4 \mathrm{~cm}$, and $x_{02}=2 \mathrm{~cm}$. From the fitting of both curves we obtain $A_{1}=41.7(0.3)$ pixels, $\omega_{01}=9.14(0.02) \mathrm{rad} / \mathrm{s}$, and $\phi_{1}=-0.91(0.02) \mathrm{rad}$ for the symmetric mode, and $A_{2}=13.8(0.3)$ pixels, $\omega_{02}=16.93(0.04) \mathrm{rad} / \mathrm{s}$, and $\phi_{2}=-$ $0.49(0.04)$ for the antisymmetric mode As it can be seen, the values of $\omega_{01}$ and $\omega_{02}$ are in very good agreement with the frequencies of the two independent normal modes obtained in section IV.A.

\section{Damped movement.}

By reducing the air flow of the air track we have a damped coupled oscillating system. Figure 6 shows the normal modes when the initial positions of the masses are $x_{01}=4 \mathrm{~cm}, x_{02}=2 \mathrm{~cm}$. From the fitting ofboth curves we obtain $A_{1}=28.5(0.6)$ pixels, $\omega_{1}=$ 9.04(0.02) $\mathrm{rad} / \mathrm{s}$, and $\phi_{1}=2.09(0.03) \mathrm{rad}$ for the symmetric mode, and $A_{2}=13.6(0.5)$ pixels, $\omega_{2}=16.84(0.04) \mathrm{rad} / \mathrm{s}$, and $\phi_{2}=-1.31(0.06)$ rad for the antisymmetric mode. The relaxation time is $\tau=4.3(0.2) \mathrm{s}$. These results agree with the theoretical predictions, as can be seen in Fig. 7; this figure that represents the position of each mass as a function of time and the corresponding linear combination of the normal modes.

\section{CONCLUDING REMARKS}

Video analysis and simple image recognition technique have proved to be a useful method for analyzing damped coupled oscillators. Furtermore, the possibility of 
following the movement frame by frame opens new ways for the understanding of complex physics process. This technique increases the pedagogical efficiency as compared to the traditional methods that require more sophisticated explanation to reach similar results.

\section{AKNOWLEDGEMENTS}

This work has received financial support by the Universidad Politécnica de Valencia (P.I.I. 20020632), Spain. We would like to thank the R+D+I Linguistic Assistance Office at the Universidad Politécnica de Valencia for their help in revising this paper. 


\section{REFERENCES}

[1] R. Fuller and D. Zollman, Physics InfoMall, Learning Team. New York (1995).

[2] H. Brasell, "The effect of real-time laboratory graphing on learning graphic representations of distance and velocity," J. Res. Sci. Teach. 24, 385-395 (1987).

[3] A. Vidaurre, J. Riera, M.H. Giménez, and J.A. Monsoriu, "Contribution of digital simulation in visualizing physics processes," Comput. Appl. Eng. Educ. 10(1), 4549 (2002).

[4] J. Riera, M.H. Giménez, A. Vidaurre, and J.A. Monsoriu, "Digital simulation of wave motion," Comput. Appl. Eng. Educ. 10(1), 161-166 (2002).

[5] L.T. Escalada and D.A. Zollman, "An investigation on the effects of using interactive digital video in a physics classroom on student learning and attitudes," J. Res. Sci. Teach. 34, 467-489 (1997).

[6] Y.-S. Shin, "Virtual reality simulations in web-based science education," Comput. Appl Eng Educ 10(1), 18-45 (2002).

[7] R.R. Cadmus, "A video technique to facilitate the visualization of physical phenomena," Am. J. Phys. 58, 397-399 (1990).

[8] R.N. Steinberg, "Computer in teaching science: To simulate or not to simulate?," Phys. Educ. Res., Am. J. Phys. Suppl. 68 (7), S37-S41 (2000).

[9] D.A. Zollman, M.L. Noble, and R. Curtin, "Modeling the motion of an athlete: An interactive video lesson for teaching physics," J. Educ. Technol. Syst. 15, 249-257 (1987).

[10] P.W. Laws, "Calculus-based physics without lectures," Phys. Today 24, 24-31 (1991).

[11] W.M. Wehrbein, "Using video analysis to investigate intermediate concepts in classical mechanics,” Am. J. Phys. 69, 818-820 (2001). 
[12] E.J. Salumbides, J. Maristela, A. Uy, and K. Karremans, “A vision-based motion sensor for undergraduate laboratories," Am. J. Phys. 70 (8), 868-871 (2002)

[13] R. Cross, "Measurements of the horizontal coefficient of restitution for a superball and a tennis ball," Am. J. Phys. 70 (5), 482-489 (2002).

[14] T. Greczylo and E. Debowska, "Using a digital video camera to examine coupled oscillations," Eur. J. Phys. 23, 441-447 (2002).

[15] H.-C. Chung, J. Liang, S. Kushiyama, and M. Shinozuka, "Digital image processing for non-linear system identification," Int. J. Non-Linear Mech. 39, 691-707 (2004).

[16] J. Riera, J.A. Monsoriu, M.H. Giménez, J.L. Hueso, and J.A.Torregrosa, "Using image recognition to automate video analysis of physical processes," Am. J. Phys. 71, 1075-1079 (2003).

[17] R. Givens, O.F.A. Bonfim, and R.B. Ormond, "Direct observation of normal modes in coupled oscillators," Am. J. Phys. 71, 87-90 (2003).

[18] A. VanderLugt, "Signal detection by complex spatial filtering" IEEE Trans. Inf. Theory IT-10, 39-145 (1964).

[19] J.D. Gaskill, Linear Systems, Fourier Transforms and Optics (John Wiley \& Sons, New York, 1978), p.196.

[20] Guide to the Expression of Uncertainty in Measurement (ISO, Switzerland, 1995). 


\section{FIGURE CAPTIONS}

Figure 1. Experimental arrangement, where the detection windows has been framed. The object to be detected is shown in the upper-right corner.

Figure 2. Correlation function between the input scene and the objects to be detected, when the distance between gliders is: (a) maximum and (b) minimum. Axes $x$ and $y$ represent pixel position in the detection window.

Figure 3. Plots of normal coordinates $\mathrm{q}_{1}$ and $\mathrm{q}_{2}$ versus time when the initial conditions favor the symmetric mode.

Figure 4. Plots of normal coordinates $\mathrm{q}_{1}$ and $\mathrm{q}_{2}$ versus time when the initial conditions favor the antisymmetric mode.

Figure 5. Plots of normal coordinates $\mathrm{q}_{1}$ and $\mathrm{q}_{2}$ versus time when there are no friction forces.

Figure 6. Plots of normal coordinates $\mathrm{q}_{1}$ and $\mathrm{q}_{2}$ versus time when damping friction forces are present.

Figure 7. Position of each mass $\left(d_{1}, d_{2}\right)$ versus time and the theoretical prediction corresponding to linear combination from the normal modes. Taking the origin in the left down corner of the window shown in Fig. 1, each mass is located in $d_{1}=166+x_{1}$ and $d_{2}=414+x_{2}$, respectively. 


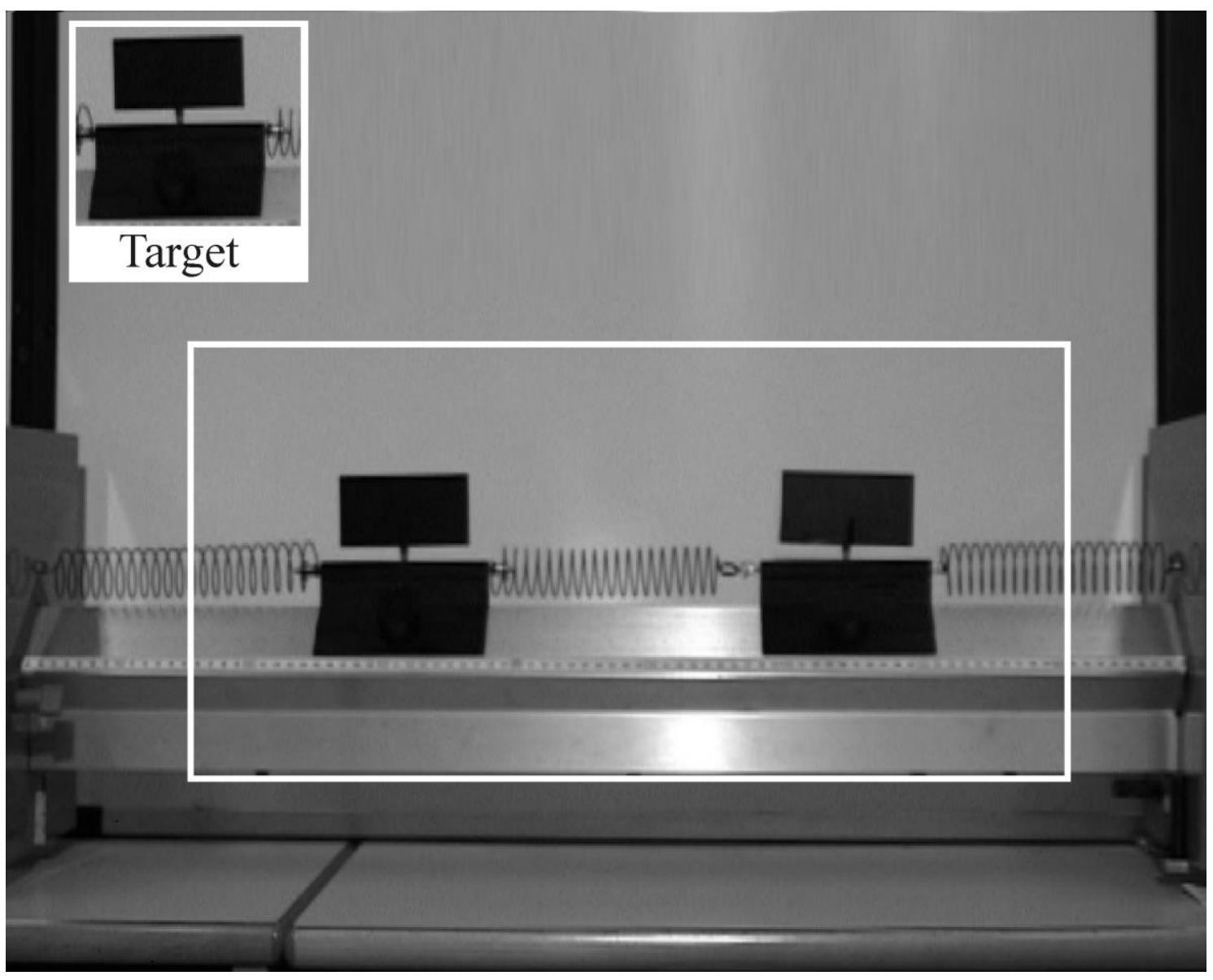

Figure 1

(J.A. Monsoriu et al.) 

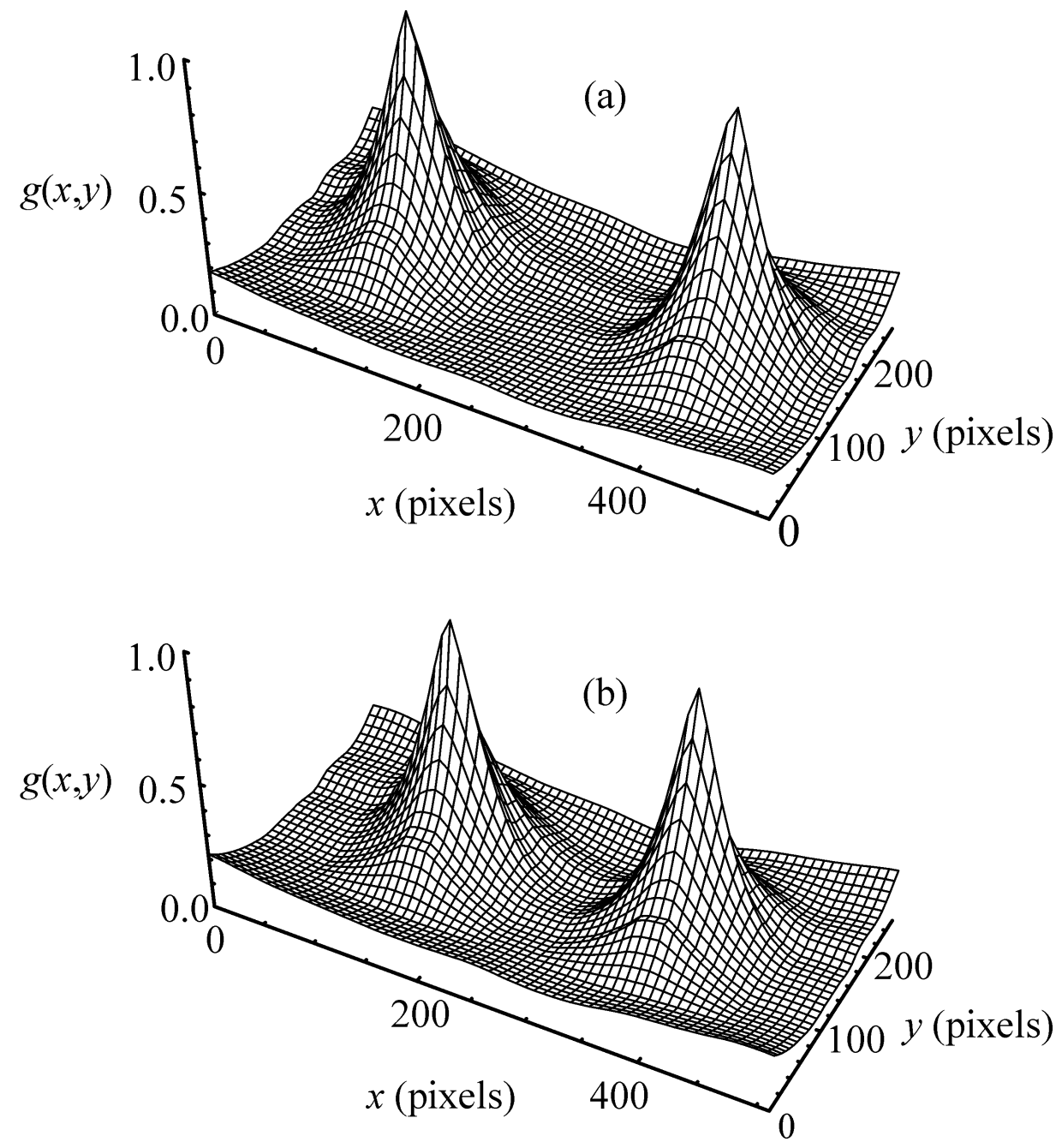

Figure 2

(J.A. Monsoriu et al.) 


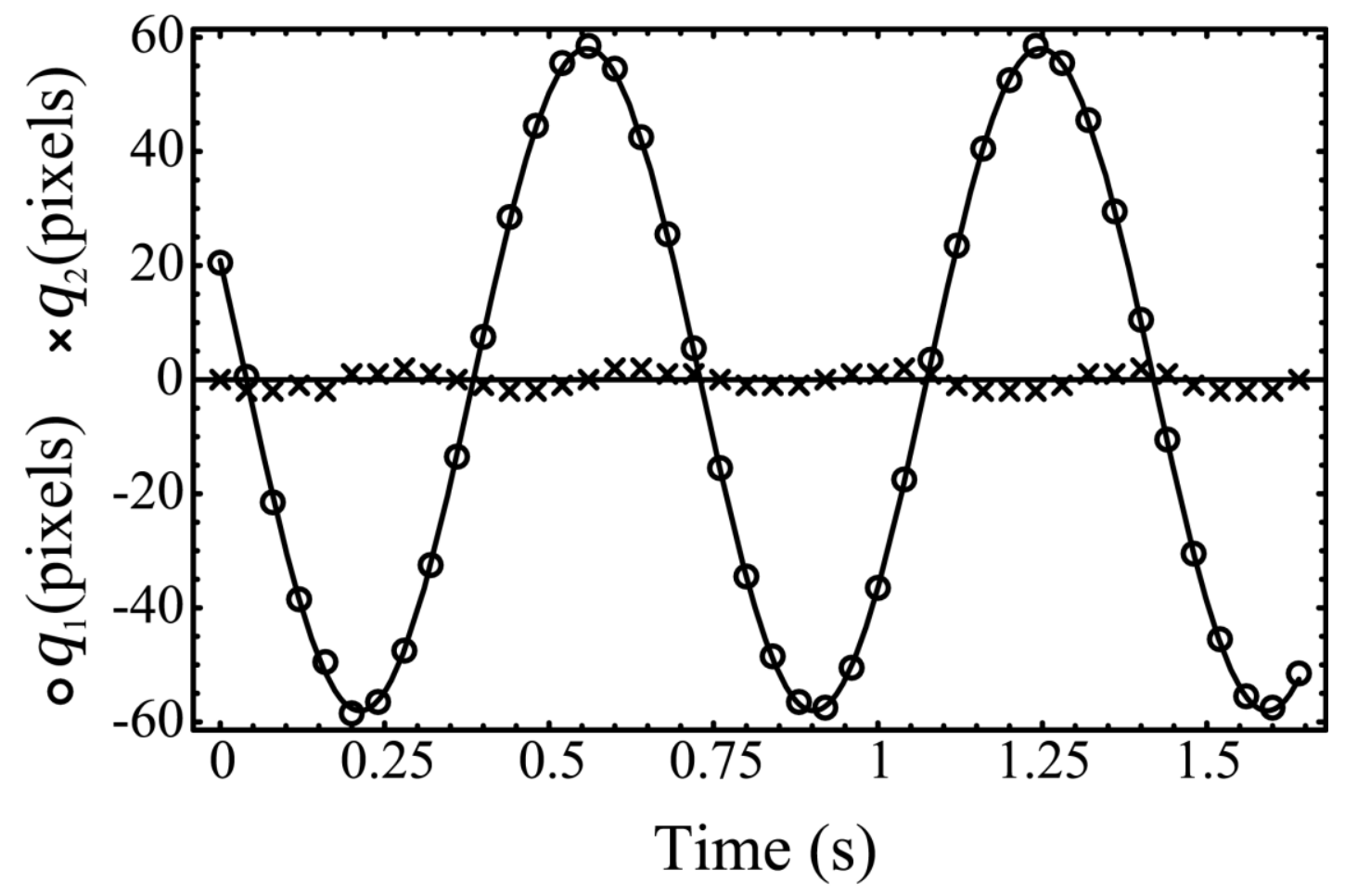

Figure 3

(J.A. Monsoriu et al.) 


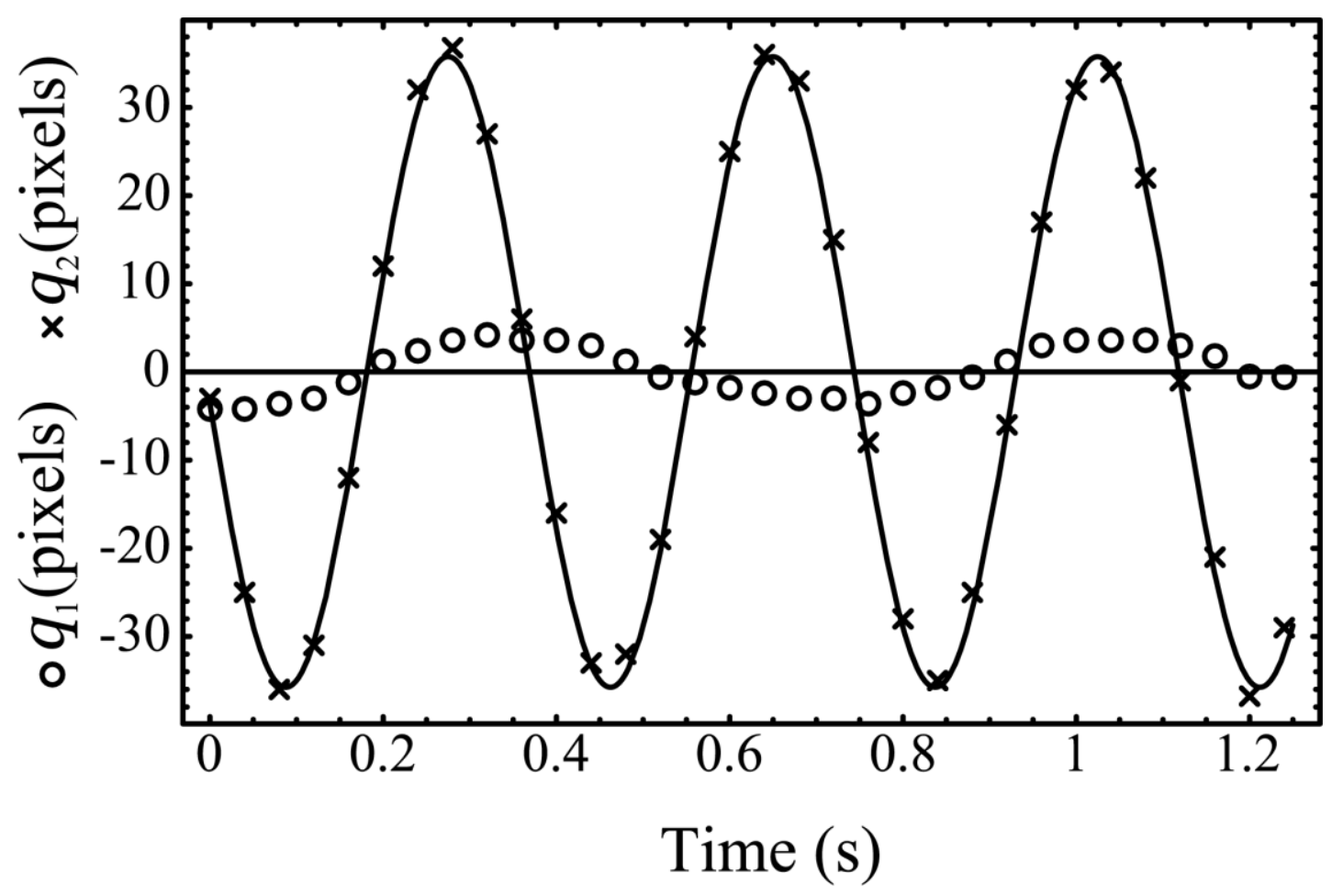

Figure 4

(J.A. Monsoriu et al.) 


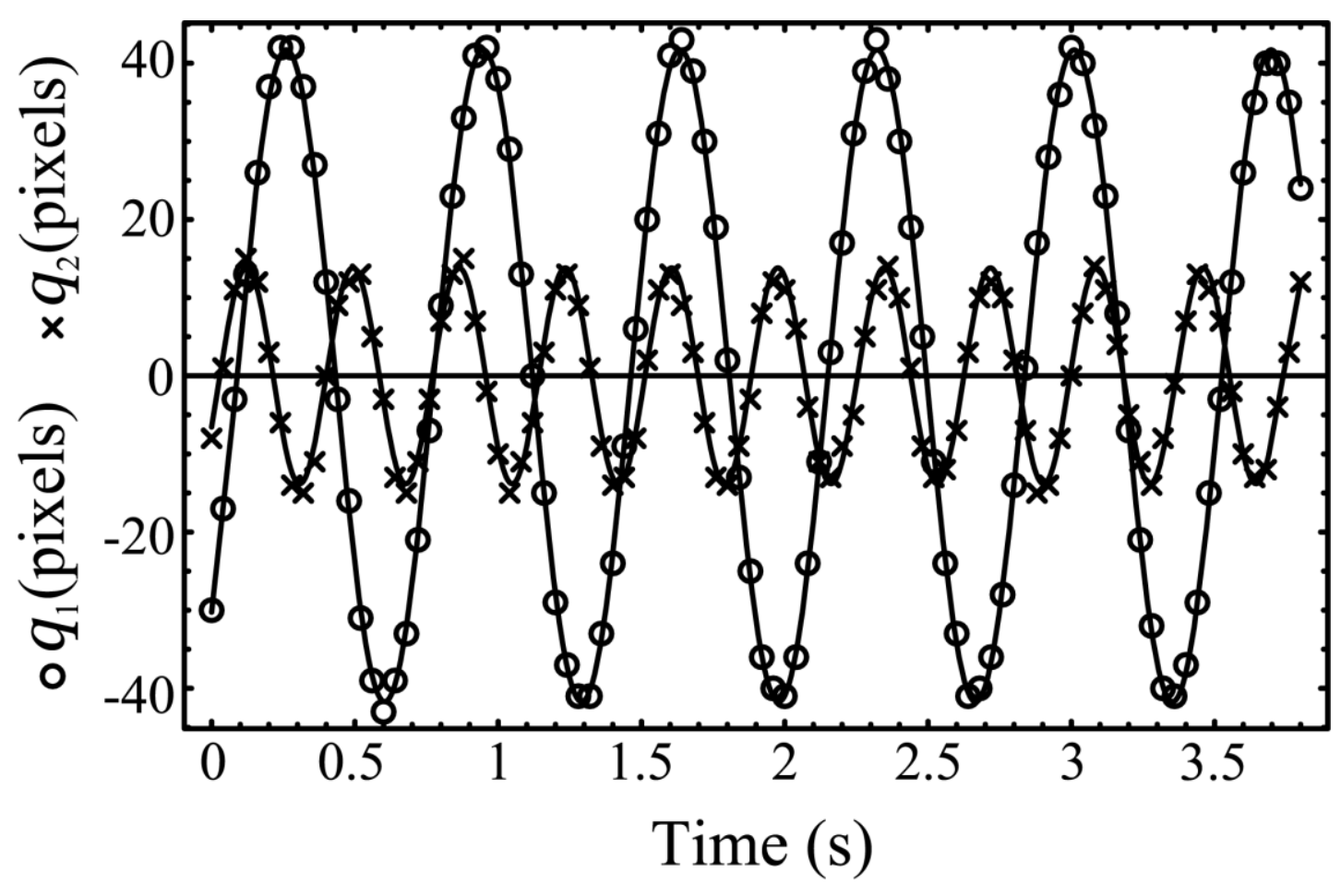

Figure 5

(J.A. Monsoriu et al.) 


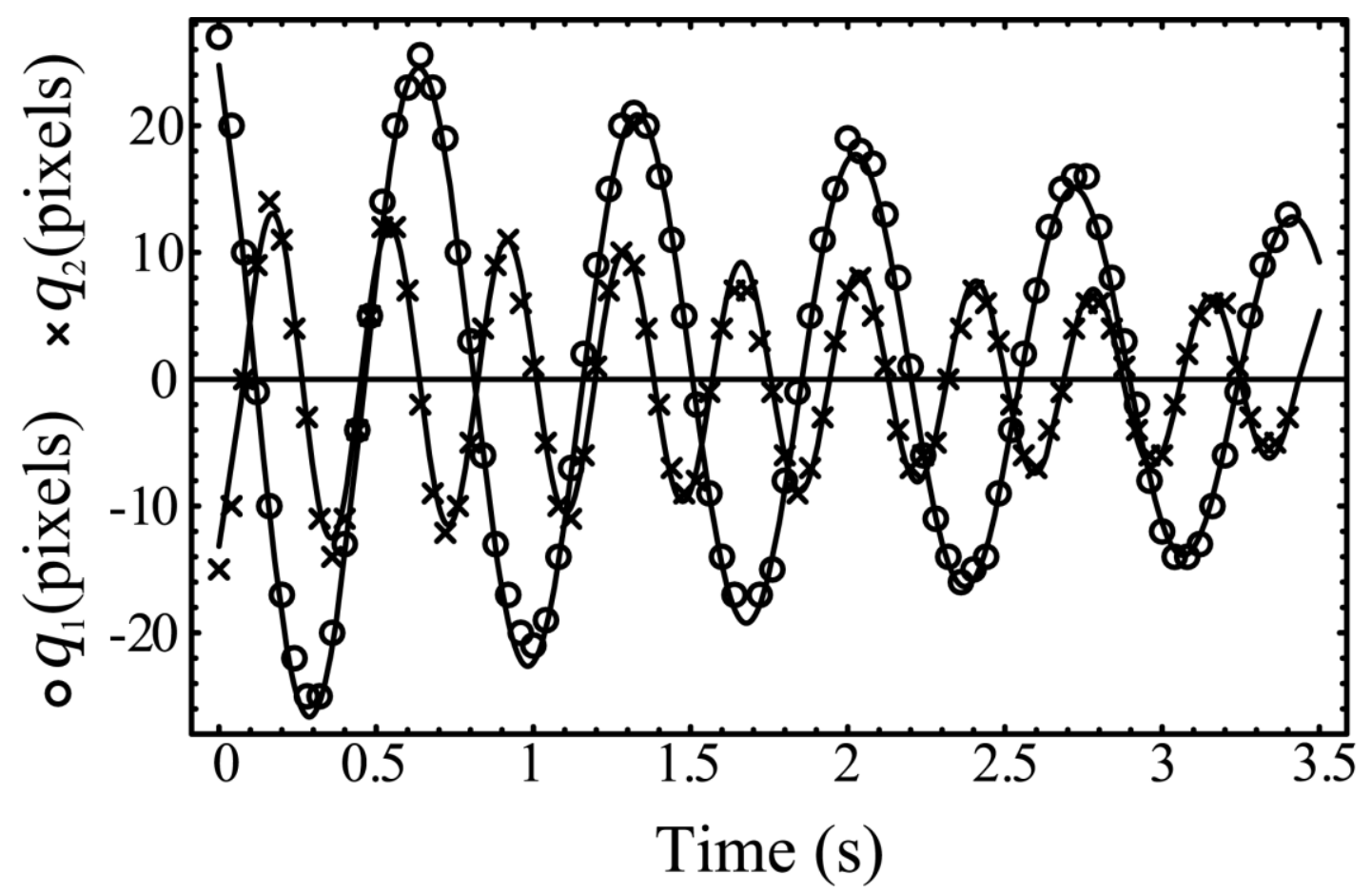

Figure 6

(J.A. Monsoriu et al.) 

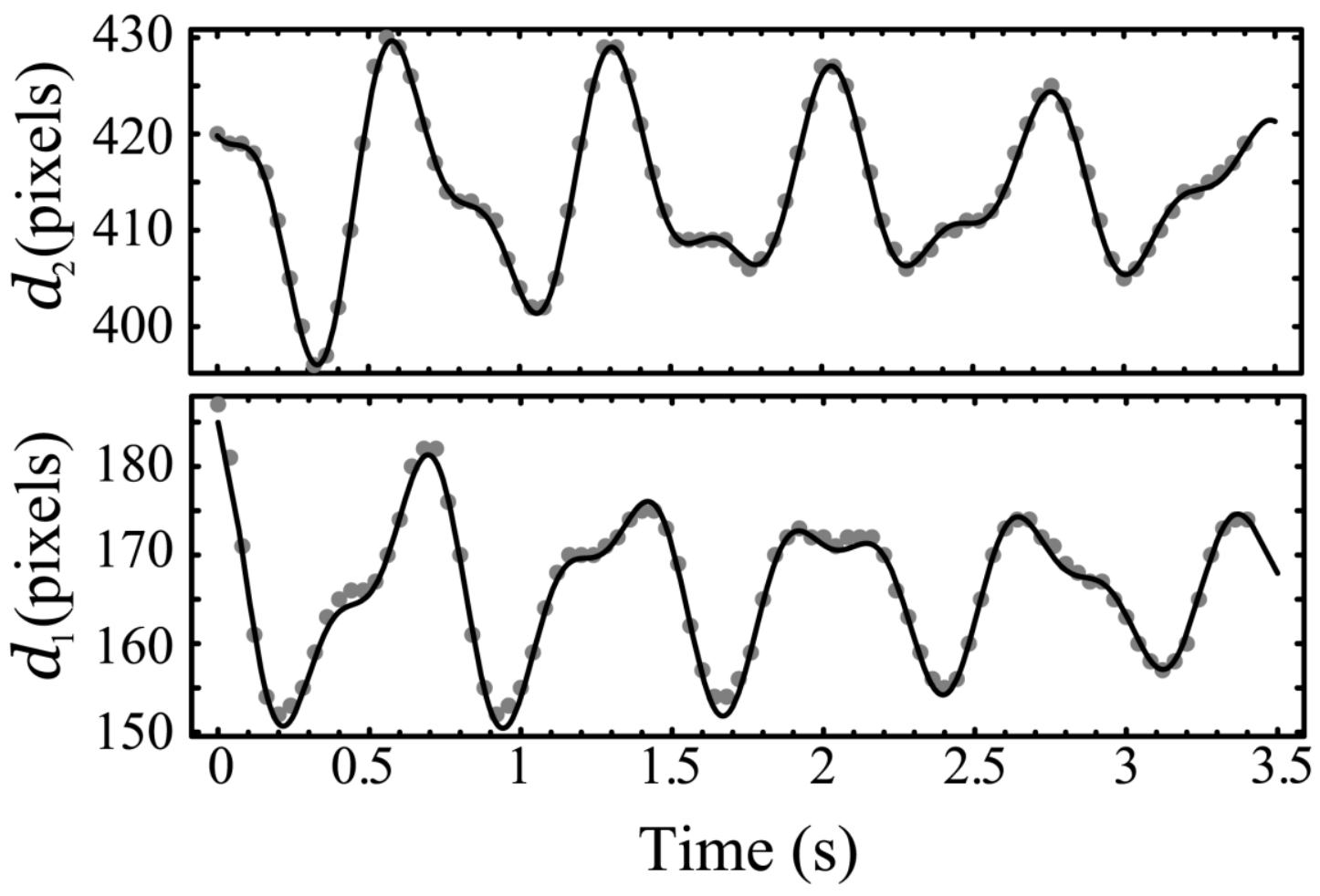

Figure 7

(J.A. Monsoriu et al.) 